\title{
Autoregressive Spectral Modeling of Motor Unit Action Potentials: Preliminary Findings
}

\author{
A. Elia ${ }^{1}$, C. Pattichis ${ }^{2}$, W. Fincham ${ }^{3}$, A. Spanias ${ }^{4}$, L. Middleton ${ }^{2}$ \\ ${ }^{1}$ Higher Technical Institute, P.O. Box 2423, Nicosia, Cyprus \\ ${ }^{2}$ The Cyprus Institute of Neurology and Genetics, P.O.Box 3462, Nicosia, Cyprus \\ ${ }^{3}$ Queen Mary and Westfield College, University of London, Mile End Road, London E1 4NS, U.K. \\ ${ }^{4}$ Arizona State University, Tempe, Arizona 85287-5706, U.S.A.
}

\begin{abstract}
Autoregressive (AR) spectral modeling of Motor Unit Action Potentials (MUAPs) has been suggested in the past but not thoroughly investigated. In this study AR spectral modeling was applied on MUAPs recorded from the biceps muscle. The AR parameters were estimated using the Yule-Walker, Burg, and Modified Covariance methods. Findings suggest that a similar spectral envelope was obtained by the Burg and the Modified Covariance methods of order 12 .
\end{abstract}

\section{INTRODUCTION}

Quantitative electromyography (EMG) is important in the diagnosis of neuromuscular disorders. In the past years a number of computer-aided methods appeared for the quantitative analysis of MUAPs. Spectral analysis of the EMG signal using classical power spectrum techniques has already been examined [1]. These methods yield power spectrum density (PSD) estimates from a windowed set of data or autocorrelation sequence estimates. The result is reduced frequency resolution and spectral leakage effects present in the spectrum. Parametric power spectrum estimation methods on the other hand are model-based techniques, and they provide better power spectral density estimators and higher resolution compared to the classical spectral estimators [2]. In clinical EMG, AR analysis was suggested as a diagnostic tool [3]. However, this method has not yet been investigated. In this work the spectral characteristics of MUAPs as obtained by using conventional power spectrum techniques and AR spectral analysis will be compared.

\section{METHOD}

\section{A. Classical Spectral Estimation}

There are two basic classical PSD estimation approaches: (i) the direct method, or periodogram, that operates directly on the data set to yield a PSD estimate, and (ii) the indirect method, or correlogram, that firstly makes an estimate of the correlation sequence, and then Fourier transforms it to obtain the PSD estimate.
In this study the Welch periodogram method will be used. This method is the most frequently used periodogram estimation approach.

\section{B. AR Spectral Modeling}

In the AR model, or the all-pole model, at time $\boldsymbol{n}$ the signal can be expressed as a linear combination of previous samples and an error signal $\boldsymbol{e}(\boldsymbol{n})$ :

$$
x(n)=-\sum_{p=1}^{k} \alpha_{p} x(n-p)+e(n)
$$

where $\quad x(n)$ is the input signal

$\alpha_{p}$ are the AR coefficients

$k \quad$ is the AR model order

$\boldsymbol{e}(n)$ is the error signal.

The AR parameters $\alpha_{p}$ 's were estimated by the following methods: (i) Yule-Walker, (ii) Burg, and (iii) Modified Covariance method.

The AR power spectral density was computed by:

$$
P_{A R}(f)=\frac{T \hat{\rho}_{p}}{\left[1+\sum_{p=1}^{k} \alpha_{p} e^{-j 2 \pi f T p}\right]^{2}}
$$

where $\hat{\rho}$ is the minimum mean-squared value of the error signal

$\boldsymbol{T}$ is the sampling period.

\section{RESULTS}

EMG was recorded from the biceps muscle for 5 seconds, bandpass filtered at $3 \mathrm{~Hz}$ to $10 \mathrm{KHz}$, and sampled at $20 \mathrm{KHz}$ with 12 bits resolution. MUAPs were 

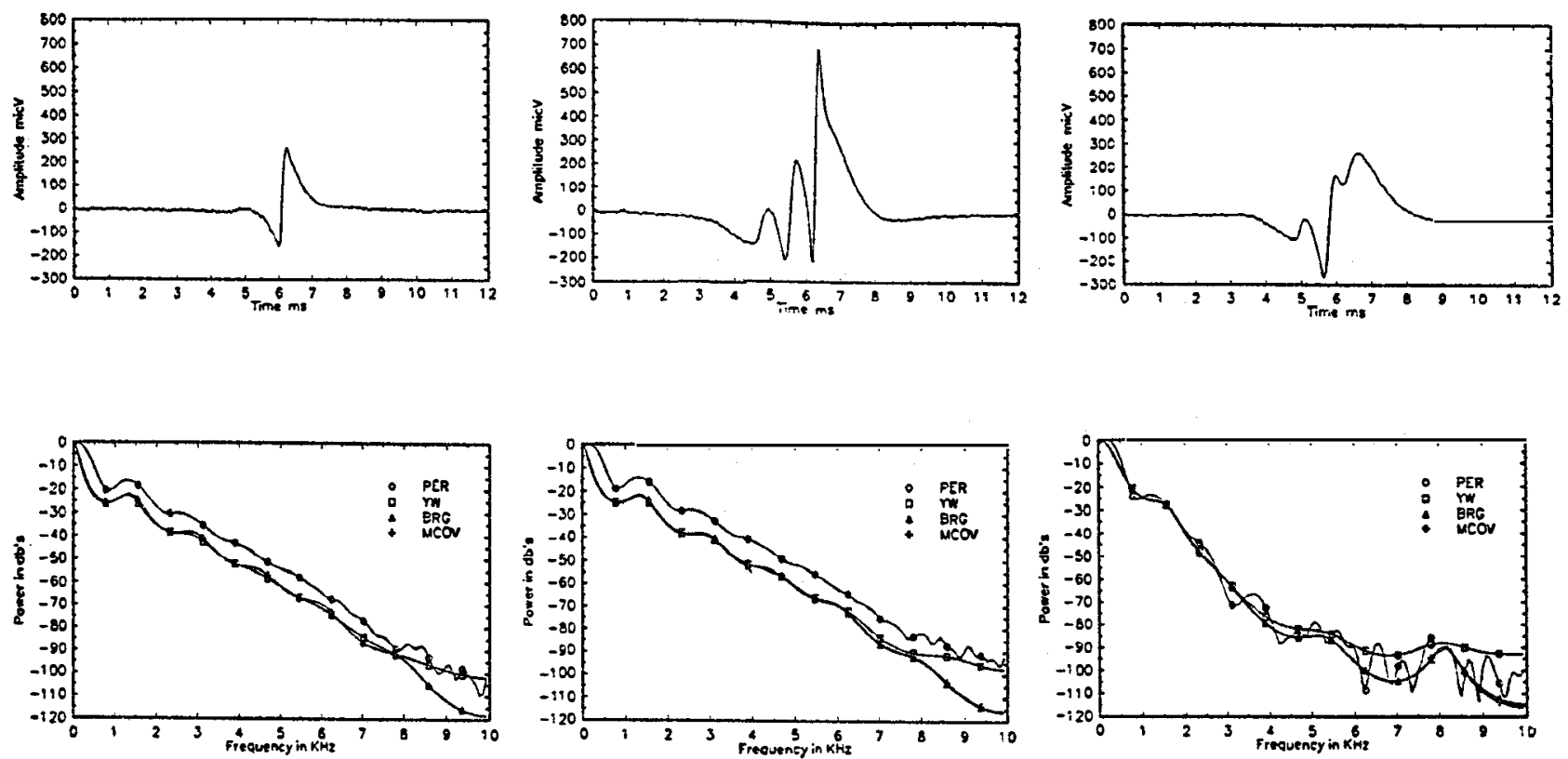

Figure 1. PSD estimates of three different MUAPs using the Periodogram (PER), Yule-Walker (YW), Burg (BRG), and Modified Covariance (MCOV) methods.

extracted, measured and classified automatically using the Parametric Pattern Recognition (PPR) technique [4]. The PPR algorithm tries to identify at least three similar MUAPs generated from the same source. MUAP parameters measured include: amplitude, duration, area, and number of phases. Traditionally at least 20 MUAP sets are collected whose parameters are averaged to obtain a diagnosis. In this study MUAP features based on AR spectral analysis were obtained.

Three different MUAP waveforms and their respective PSD estimates are given in Figure 1. A data sequence of 128 points $(6.4 \mathrm{~ms})$ with the mean removed was used to produce the PSD estimates. In the Welch periodogram method the Hann window with $50 \%$ overlap of the segments was implemented. Therefore, the periodogram used three segments of 64 sample-intervals and 32 samples overlap. In AR analysis the Final Prediction Error (FPE), the Akaike Information Criterion (AIC), and the Minimum Description Length (MDL) criteria for model order selection were computed [2]. These criteria gave an order in the range of 410. However, AR models of order 12 were implemented as this order seemed to give satisfactory PSD estimates.

As it is shown in Figure 1, PSD estimates obtained showed that most of the power in MUAPs is concentrated at low frequencies. The Welch periodogram has a lower resolution compared to the AR methods. The Yule-Walker method produced AR spectra with lower resolution compared to the other two AR methods. The Burg and the Modified Covariance methods produced comparable AR spectral estimates.

Although these findings are preliminary, it seems that AR spectral modeling provides useful information for EMG diagnosis. Future work will be carried out on MUAPs recorded from patients with motor neuron disease (MND) and myopathy. Finally, it is suggested that $\mathrm{AR}$ features can be combined with time domain features to build an automated EMG diagnostic system using neural networks.

\section{REFERENCES}

[1] $H$. Ide, and S. Obata, "Feature characterisation of shape from the frequency spectrum of EMG", Med. and Biol. and Comp. 21:66-171, 1983.

[2] S. L. Marple Jr., Digital Spectral Analysis with Applications, Prentice-Hall, USA, 1987.

[3] J. V. Basmajian, et al., "Electrodiagnostic Model for Motor Unit Action Potential (MUAP) Generation", American Journal of Phys. Medic., Vol 64, No 6, 1985. [4] C.S. Pattichis, Artificial Neural Networks in Clinical Electromyography, Ph.D. Thesis, Univ. of London, 1992. 